\begin{tabular}{|c|c|}
\hline Title & $\begin{array}{l}\text { Synthesis of 1,1-Spirobiindane-7,7-Disulfonic A cid and Disulfonimide: A pplication for Catalytic A symmetric } \\
\text { A minalization }\end{array}$ \\
\hline Author(s) & Kurihara, T akumaru; Satake, Shun; Hatano, Manabu; Ishihara, Kazuaki; Y oshino, T atsuhiko; Matsunaga, Shigeki \\
\hline Citation & $\begin{array}{l}\text { Chemistry-an asian journal, 13(17), 2378-2381 } \\
\text { https://doi.org/10.1002/asia.201800341 }\end{array}$ \\
\hline Issue Date & 2018-09-04 \\
\hline Doc URL & http:/hdl.handle.net/2115/75353 \\
\hline Rights & $\begin{array}{l}\text { This is the peer reviewed version of the following article: } \\
\text { https://onlinelibrary.wiley .com/doi/abs/10.1002/asia.201800341, which has been published in final form at } \\
10.1002 / \text { asia.201800341. This article may be used for non-commercial purposes in accordance with Wiley Terms and } \\
\text { Conditions for Self-A rchiving }\end{array}$ \\
\hline Type & article (author version) \\
\hline File Information & WoS_85746_Y oshino.pdf \\
\hline
\end{tabular}

Instructions for use 


\title{
Synthesis of 1,1'-Spirobiindane-7,7'-Disulfonic Acid and Disulfonimide: Application for Catalytic Asymmetric
}

\section{Aminalization}

\author{
Takumaru Kurihara,${ }^{[\mathrm{a}]}$ Shun Satake,${ }^{[\mathrm{a}]}$ Manabu Hatano,${ }^{[\mathrm{b}]}$ Kazuaki Ishihara,${ }^{[\mathrm{b}]}$ Tatsuhiko Yoshino, ${ }^{* \mathrm{a}]}$ and \\ Shigeki Matsunaga*[a]
}

\begin{abstract}
Spirobiindane-7,7'-disulfonic acid (SPISA) and 1,1'spirobiindane-7,7'-disulfonimide were synthesized from 1,1'spirobiindane-7,7'-diol (SPINOL) in 4 steps using Pd-catalyzed Newman-Kwart rearrangement as a key step. These new catalysts possessing a rigid spirocyclic backbone were evaluated in a catalytic asymmetric aminalization reaction, and SPISA/iPr ${ }_{2} \mathrm{NEt}$ exhibited high enantioselectivity, demonstrating the utility of SPISA as a chiral Brønsted acid catalyst.
\end{abstract}

Chiral Brønsted acid catalysts are powerful tools for the stereoselective construction of $\mathrm{C}-\mathrm{C}$ and $\mathrm{C}$-hetero atom bonds in modern organic synthesis. ${ }^{[1]}$ Among a variety of catalysts reported to date, chiral 1,1'-binaphthyl-2,2'-disulfonic acids (BINSAs) and their disulfonimide derivatives (Figure 1, top ${ }^{[2,3]}$ are attractive in terms of their stronger Brønsted acidity compared with chiral phosphoric acids and carboxylic acids. ${ }^{[1]}$ In addition to working as a Brønsted acid, these catalysts can be used as ligands for a metal center ${ }^{[3 b]}$ and chiral anions to control enantioselectivity. ${ }^{[3, e, k, 1,4]}$ Their versatility and efficient catalytic activity have prompted chemists to pursue the steric and electronic tuning of these catalysts. ${ }^{[3 \mathrm{c}, \mathrm{f}, \mathrm{g}, j]}$ In general, catalytic asymmetric synthesis often requires fine-tuning of the catalyst to achieve high enantioselectivity with specific substrates, and increasing the diversity of available catalyst structures is crucial.

Here we report the synthesis of 1,1'-spirobiindane-7,7'disulfonic acid (SPISA) 1 and 1,1'-spirobiindane-7,7'disulfonimide 2 (Figure 1, bottom). The 1,1'-spirobiindane structure is an axially chiral backbone that is more rigid and less flexible than a 1,1'-binaphthyl backbone. The rigidity is sometimes beneficial in asymmetric catalysis because the conformational ambiguity in an enantio-determining step can be omitted. A variety of chiral ligands were synthesized from 1,1'spirobiindane-7,7'-diol (SPINOL) 3 by Zhou ${ }^{[5-8]}$ and others, ${ }^{[9,10]}$ and exhibited excellent

[a] T. Kurihara, S. Satake, Dr. T. Yoshino, Prof. Dr. S. Matsunaga Faculty of Pharmaceutical Sciences Hokkaido University

Kita-12 Nishi-6, Kita-ku, Sapporo 060-0812 (Japan)

E-mail: tyoshino@pharm.hokudai.ac.jp;

smatsuna@pharm.hokudai.ac.jp

[b] Dr. M. Hatano, Prof. Dr. K. Ishihara

Graduate School of Engineering

Nagoya University

Furo-cho, Chikusa, Nagoya 464-8603 (Japan)

Supporting information for this article is given via a link at the end of the document.
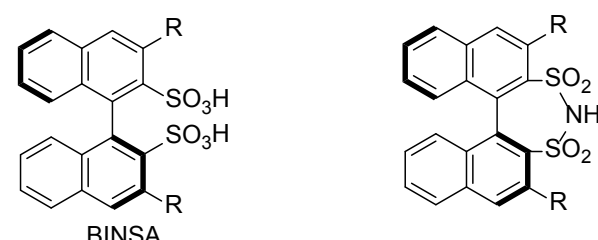

BINSA

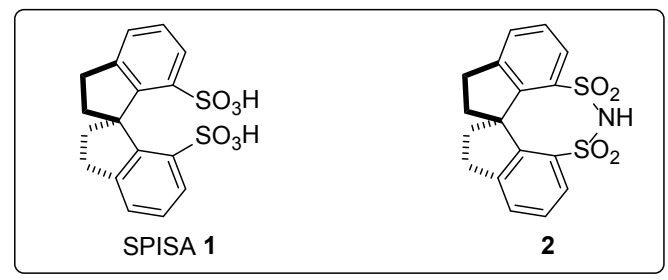

Figure 1. Structures of BINSA (1,1'-binaphthyl-2,2'-disulfonic acid), SPISA (1,1'-spirobiindane-7,7'-disulfonic acid), and their disulfonimide derivatives

enantioselectivity in transition metal-catalyzed reactions Although chiral phosphoric acids possessing the spirocyclic core were also reported, ${ }^{[11]}$ syntheses of SPISA 1 and sulfonimide 2 have not yet been reported. ${ }^{[12]}$

We synthesized 1 and 2 from (R)-SPINOL 3 using a route similar to that used for BINSA (Scheme 1). ${ }^{[3 a, 3 d, 13,14]}$ Thiocarbamoylation of the phenolic hydroxy groups of $\mathbf{3}$ under the reported conditions for BINOL $\left(\mathrm{NaH}, \mathrm{Me}_{2} \mathrm{NC}(\mathrm{S}) \mathrm{Cl}\right.$, DMF, $\left.85^{\circ} \mathrm{C}\right)^{[3 \mathrm{a}, 3 \mathrm{~d}]}$ resulted in poor conversion, but changing the base to $\mathrm{DABCO}^{[15]}$ significantly enhanced the reactivity, providing 4 in $81 \%$ at room temperature. With the precursor for key NewmanKwart rearrangement (NKR) in hand, we investigated several conditions for NKR as shown in the table in Scheme 1. ${ }^{[16]}$ Although thermal conditions, which are usually used for the synthesis of BINSA, afforded a complex mixture, Pd-catalyzed NKR reported by Lloyd-Jones et al. ${ }^{[16 b]}$ furnished the desired product 5 in $86 \%$ yield. We also tried NKR under photo-redox catalysis using 6 reported by Nicewicz et al., ${ }^{[16 c]}$ but the reaction barely occurred. Oxidation of $\mathbf{5}$ using $\mathrm{N}$-chlorosuccinimide (NCS) in $\mathrm{MeOH} / \mathrm{THF}$ smoothly proceeded to give disulfonyl chloride 7 , and subsequent hydrolylsis and protonation afforded $(R)$-SPISA 1. Disulfonimide 2 was also obtained in $89 \%$ yield by treating 7 with gasous ammonia.

To evaluate the catalytic activity as a Brønsted acid as well as the stereoinduction efficiency of $\mathbf{1}$ and $\mathbf{2}$, we selected catalytic asymmetric aminalization of Cbz-protected imines with 


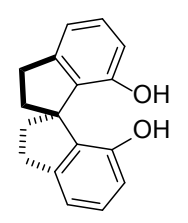

(R)-SPINOL 3
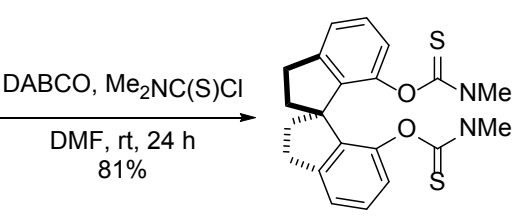

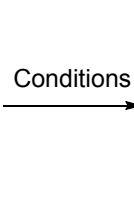

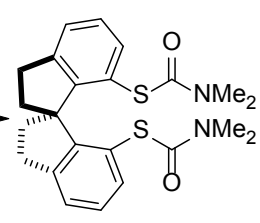

5

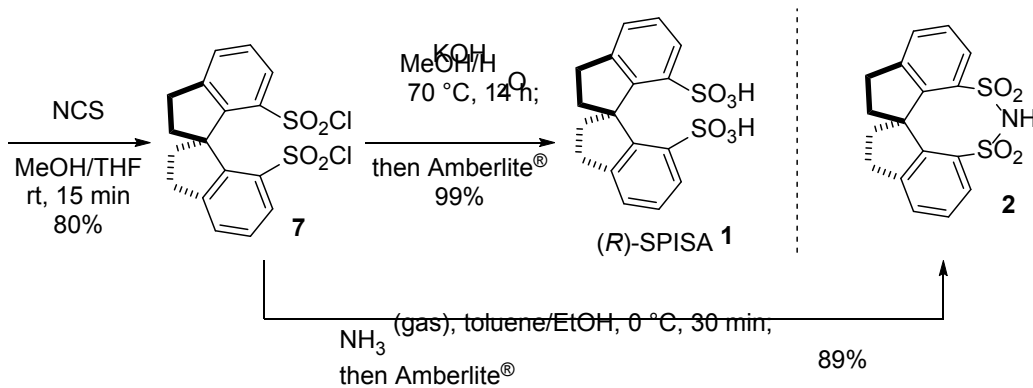

\begin{tabular}{ll}
\hline Conditions & Results \\
\hline $\begin{array}{l}\text { 1,2,4-trichlorobenzene } \\
230{ }^{\circ} \mathrm{C}, 24 \mathrm{~h}\end{array}$ & decomp. \\
& \\
$\mathrm{Pd}\left(\mathrm{PtBu}_{3}\right) 2,(8 \mathrm{~mol} \%)$ & $86 \%$ \\
toluene $, 105{ }^{\circ} \mathrm{C}, 3 \mathrm{~h}$ &
\end{tabular}

toluene, $105^{\circ} \mathrm{C}, 3 \mathrm{~h}$

$86 \%$

Blue LED

then Amberlite ${ }^{\circledR}$

Scheme 1. Synthesis of (R)-SPISA 1 and disulfonimide 2 from (R)-SPINOL 3.

carboxamides, which were previously reported using BINSA, ${ }^{[3 i, 17]}$ as a model reaction. Optimization of the reaction conditions using $\mathbf{8 a}$ and $\mathbf{9 a}$ is summarized in Table 1. The use of $\mathbf{1}$ or $\mathbf{2}$ as a sole catalyst promoted the reaction in the presence of $\mathrm{MgSO}_{4}$ as desiccant in $\mathrm{CH}_{2} \mathrm{Cl}_{2}$ at $0{ }^{\circ} \mathrm{C}$, but low enantioselectivities were observed (entries 1 and 2). We next investigated the effect of amine additives using disulfonic acid 1 as the catalyst. Formation of the mono ammonium salt of $\mathbf{1}$ was expected to improve the enantioselectivity because the ammonium moiety can increase steric bulkiness around the catalytic site without introducing ortho-substituents. ${ }^{[3,18]}$ Screening of various amines (entries 3-8) revealed that $\mathrm{Pr}_{2} \mathrm{NEt}$ was the best additive affording 10 aa in $84 \%$ ee. In the previous report using BINSA, ${ }^{[3 i]}$ 2,6-diarylpyridines were effective additives in terms of enantioselectivity. In contrast, bulky trialkylamines were clearly better than $2,6-\mathrm{Ph}_{2}$-pyridine (entry $3,14 \%$ ee) in this case, indicating that the chiral environment of SPISA 1 differs somewhat from that of BINSA. Although neither changing the solvent nor changing the dessicant further improved the result (entries 9-13), ${ }^{[19]}$ higher enantioselectivity was obtained by performing the reaction at $-40{ }^{\circ} \mathrm{C}$ for $12 \mathrm{~h}$ (entry 14). Under the optimized conditions, 10 aa was obtained in $75 \%$ yield and $93 \%$ $e$ e. $^{[20]}$

The substrate scope of the (R)-SPISA 1-catalyzed enantioselective aminalization under the optimized conditions is shown in Scheme 2. The substituent of the aromatic carboxamides had almost no effect on the enantioselectivity, and the products were obtained in moderate to good yield and with high enantioselectivity (10aa-10ac). ${ }^{[21]}$ Acrylamide $9 \mathrm{~d}$ was also compatible under the same conditions, although the enantioselectivity was slightly diminished (10ad). In addition to imine $\mathbf{8 a}$, both electron-rich and electron-deficient imines gave high enantioselectivity (10ba-10bc, 10ca).

In summary, we synthesized (R)-SPISA 1 and disulfonimide 2 in 4 steps from $(R)$-SPINOL 3 using Pd-catalyzed NewmanKwart rearrangement as a key step. These new chiral Brønsted acid catalysts were evaluated in the catalytic asymmetric aminalization of imines $\mathbf{8}$ and carboxamides $\mathbf{9}$, and the combination of 1 and $\mathrm{Pr}_{2} \mathrm{NEt}$ exhibited high enantioselectivity. These catalysts can be a useful and good option for the development of asymmetric Brønsted acid catalysis as well as asymmetic counteranion-directed catalysis. ${ }^{[4]}$ Further application of $(R)$-SPISA 1 and disulfonimide 2 as well as their structural tuning at the 6,6 '-positions are ongoing.

Table 1. Optimization for enantioselective aminalization. ${ }^{[a]}$

\begin{tabular}{|c|c|c|c|c|c|c|}
\hline \multirow[b]{2}{*}{ Entry } & \multirow{2}{*}{$\begin{array}{l}8 \mathbf{a} \\
\text { Catalyst }\end{array}$} & \multirow{2}{*}{ Amine } & \multirow{2}{*}{$\begin{array}{c}\begin{array}{c}\text { catalyst }(5 \mathrm{~mol} \% \\
\text { amine }(5 \mathrm{~mol} \%) \\
\text { desiccant }\end{array} \\
\text { solvent, } 0^{\circ} \mathrm{C}, 1 \mathrm{~h} \\
\text { Desiccant }\end{array}$} & \multicolumn{3}{|c|}{ 6) $\underset{\substack{\text { 10aa } \\
\mathrm{Ar}=4-\mathrm{MeO}-\mathrm{C}_{6} \mathrm{H}_{4}}}{\mathrm{Cbz}} \underbrace{\mathrm{NH}}_{\mathrm{Ar}}$} \\
\hline & & & & Solvent & $\%$ Yield $^{[b]}$ & $\% e e^{[c]}$ \\
\hline 1 & 1 & none & $\mathrm{MgSO}_{4}$ & $\mathrm{CH}_{2} \mathrm{Cl}_{2}$ & 82 & $7^{[\mathrm{d}]}$ \\
\hline 2 & 2 & none & $\mathrm{MgSO}_{4}$ & $\mathrm{CH}_{2} \mathrm{Cl}_{2}$ & 88 & 31 \\
\hline 3 & 1 & $\begin{array}{l}2,6-\mathrm{Ph}_{2-} \\
\text { pyridine }\end{array}$ & $\mathrm{MgSO}_{4}$ & $\mathrm{CH}_{2} \mathrm{Cl}_{2}$ & 84 & 14 \\
\hline 4 & 1 & $\mathrm{Me}_{2} \mathrm{NBu}$ & $\mathrm{MgSO}_{4}$ & $\mathrm{CH}_{2} \mathrm{Cl}_{2}$ & $>95$ & 66 \\
\hline 5 & 1 & $\mathrm{Me}_{2} \mathrm{NCy}$ & $\mathrm{MgSO}_{4}$ & $\mathrm{CH}_{2} \mathrm{Cl}_{2}$ & 49 & 73 \\
\hline 6 & 1 & $\mathrm{Bu}_{3} \mathrm{~N}$ & $\mathrm{MgSO}_{4}$ & $\mathrm{CH}_{2} \mathrm{Cl}_{2}$ & 42 & 75 \\
\hline 7 & 1 & $\mathrm{Cy}_{2} \mathrm{NMe}$ & $\mathrm{MgSO}_{4}$ & $\mathrm{CH}_{2} \mathrm{Cl}_{2}$ & 64 & 83 \\
\hline 8 & 1 & $\mathrm{PPr}_{2} \mathrm{NEt}$ & $\mathrm{MgSO}_{4}$ & $\mathrm{CH}_{2} \mathrm{Cl}_{2}$ & 75 & 84 \\
\hline 9 & 1 & ${ }_{i P r} \mathrm{NEt}$ & $\mathrm{MgSO}_{4}$ & toluene & 54 & 67 \\
\hline 10 & 1 & $\mathrm{PPr}_{2} \mathrm{NEt}$ & $\mathrm{MgSO}_{4}$ & THF & 58 & 43 \\
\hline 11 & 1 & $i \mathrm{Pr}_{2} \mathrm{NEt}$ & $\mathrm{MgSO}_{4}$ & DCE & 68 & 75 \\
\hline 12 & 1 & $i \mathrm{Pr}_{2} \mathrm{NEt}$ & MS4A & $\mathrm{CH}_{2} \mathrm{Cl}_{2}$ & 70 & 79 \\
\hline 13 & 1 & $i \mathrm{Pr}_{2} \mathrm{NEt}$ & Drierite $^{\circledR}$ & $\mathrm{CH}_{2} \mathrm{Cl}_{2}$ & 81 & 83 \\
\hline $14^{[\mathrm{e}]}$ & 1 & $i \mathrm{Pr}_{2} \mathrm{NEt}$ & $\mathrm{MgSO}_{4}$ & $\mathrm{CH}_{2} \mathrm{Cl}_{2}$ & 75 & 93 \\
\hline
\end{tabular}

[a] Reactions were run using 8 a $(0.30 \mathrm{mmol}), 9 a(0.20 \mathrm{mmol})$, catalyst $(0.01 \mathrm{mmol}$, $5 \mathrm{~mol} \%$ ), amine $(0.01 \mathrm{mmol}, 5 \mathrm{~mol} \%)$, and $\mathrm{MgSO}_{4}(0.34 \mathrm{mmol})$, MS4A (40 mg), or Drierite $^{\circledR}(40 \mathrm{mg})$ in solvent $(1.0 \mathrm{~mL})$ at $0{ }^{\circ} \mathrm{C}$ for $1 \mathrm{~h}$ unless otherwise noted. [b] Isolated yield. [c] Determined by chiral HPLC analysis. See Supporting Information for more details. [d] The opposite enantiomer was obtained as a major product. [e] $-40{ }^{\circ} \mathrm{C}, 12 \mathrm{~h}$. 


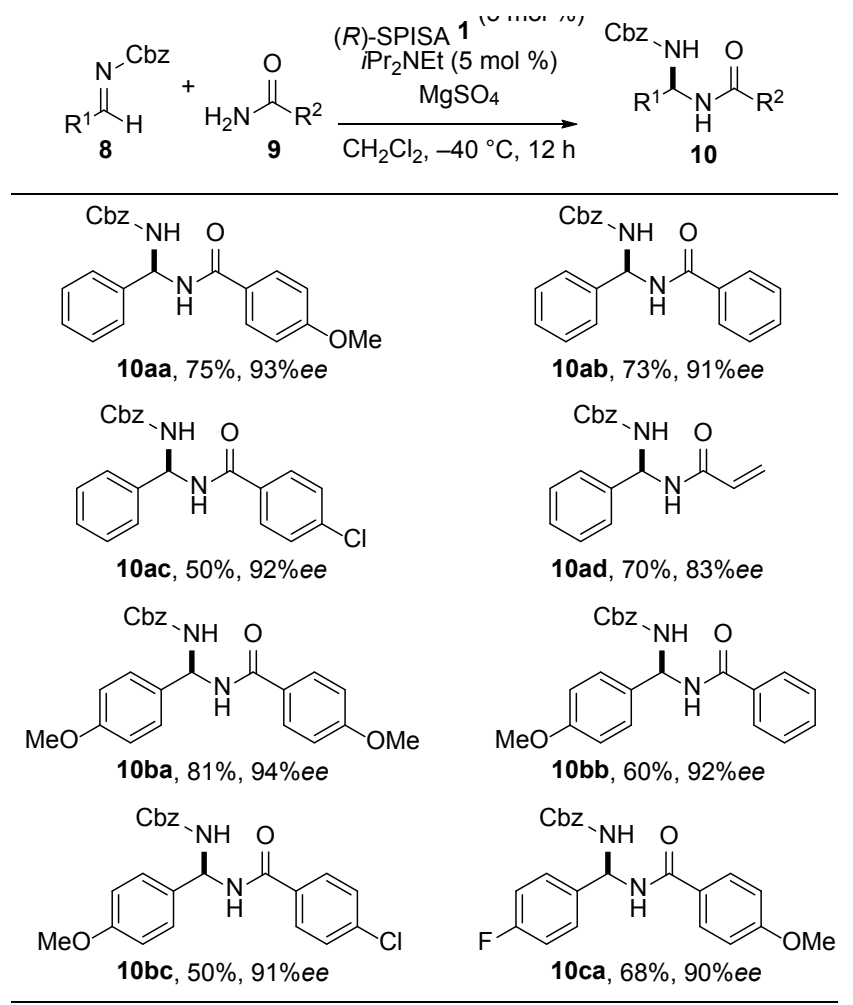

Scheme 2. Substrate scope of (R)-SPISA 1-catalyzed asymmetric aminalization.

\section{Acknowledgements}

This work was supported in part by JSPS KAKENHI Grant Number JP15H05802 and $15 \mathrm{H} 05810$ in Precisely Designed Catalysts with Customized Scaffolding, JSPS KAKENHI Grant Number JP17K15417, and The Uehara Memorial Foundation (S.M.).

Keywords: chiral Brønsted acid $・ 1,1$ '-spirobiindane $\cdot$ disulfonic acid $\cdot$ disulfonimide $\cdot$ aminalization

[1] a) T. Akiyama, Chem. Rev. 2007, 107, 5744-5758; b) M. Terada Synthesis 2010, 1929-1982; c) T. Hashimoto, K. Maruoka, J. Synth. Org. Chem. Jpn. 2013, 71, 472-479; d) D. Parmar, E. Sugiono, S. Raja, M. Rueping, Chem. Rev. 2014, 114, 9047-9153; e) T. Akiyama, K. Mori, Chem. Rev. 2015, 115, 9277-9306; f) C. Min, D. Seidel, Chem. Soc. Rev. 2017, 46, 5889-5902.

[2] Reviews on chiral BINSAs and its disulfonimides in asymmetric catalysis: a) M. Hatano, K. Ishihara, Asian J. Org. Chem. 2014, 3, 352365; b) T. James, M. van Gemmeren, B. List, Chem. Rev. 2015, 115, 9388-9409.

[3] a) M. Hatano, T. Maki, K. Moriyama, M. Arinobe, K. Ishihara, J. Am. Chem. Soc. 2008, 130, 16858-16860; b) M. Hatano, Y. Hattori, Y. Furuya, K. Ishihara, Org. Lett. 2009, 11, 2321-2324; c) P. GarcíaGarcía, F. Lay, P. García-García, C. Rabalakos, B. List, Angew. Chem. 2009, 121, 4427-4430; Angew. Chem. Int. Ed. 2009, 48, 4363-4366; d) M. Treskow, J. Neudörfl, R. Giernoth, Eur. J. Org. Chem. 2009, 36933697 ; e) R. L. Lalonde, Z. J. Wang, M. Mba, A. D. Lackner, F. D. Toste, Angew. Chem. 2010, 122, 608-611; Angew. Chem. Int. Ed. 2010, 49, 598-601; f) M. Hatano, Y. Sugiura, K. Ishihara, Tetrahedron: Asymmetry 2010, 21, 1311-1314; g) H. He, L.-Y. Chen, W.-Y. Wong, W.-H. Chan, A. W. M. Lee, Eur. J. Org. Chem. 2010, 4181-4184; h) L.Y. Chen, H. He, W.-H. Chan, A. W. Lee, J. Org. Chem. 2011, 76, 71417147 ; i) M. Hatano, T. Ozaki, Y. Sugiura, K. Ishihara, Chem. Commun.
2012, 48, 4986-4988; j) M. Hatano, T. Ozaki, K. Nishikawa, K. Ishihara, J. Org. Chem. 2013, 78, 10405-10413; k) Q. Wang, M. Leutzsch, M van Gemmeren, B. List, J. Am. Chem. Soc. 2013, 135, 15334-15337; I) Z. Zhang, H. Y. Bae, J. Guin, C. Rabalakos, M. van Gemmeren, M. Leutzsch, M. Klussmann, B. List, Nat. Commun. 2016, 7, 12478; m) M. Hatano, K. Nishikawa, K. Ishihara, J. Am. Chem. Soc. 2017, 139, 84248427 ; n) M. Hatano, T. Mochizuki, K. Nishikawa, K. Ishihara, ACS Catal. 2018, 8, 349-353.

[4] Reviews on Asymmetric Counteranion-Directed Catalysis (ACDC): a) $R$ J. Phipps, G. L. Hamilton, F. D. Toste, Nat. Chem. 2012, 4, 603-614; b) M. Mahlau, B. List, Angew. Chem. 2013, 125, 540-556; Angew. Chem. Int. Ed. 2013, 52, 518-533.

[5] a) J. H. Xie, Q.-L. Zhou, Acc. Chem. Res. 2008, 41, 581-593; b) S. F. Zhu, Q.-L. Zhou, Acc. Chem. Res. 2012, 45, 1365-1377; c) S. F. Zhu, Q.-L. Zhou, Acc. Chem. Res. 2017, 50, 988-1001.

[6] Monodentate and bidentate phosphorus ligands: a) A. G. Hu, Y. Fu, J.H. Xie, H. Zhou, L.-X. Wang, Q.-L. Zhou, Angew. Chem. 2002, 114, 2454-2456; Angew. Chem. Int. Ed. 2002, 41, 2348-2350; b) J.-H. Xie L.-X. Wang, Y. Fu, S.-F. Zhu, B.-M. Fan, H.-F. Duan, Q.-L. Zhou, J. Am. Chem. Soc. 2003, 125, 4404-4405; c) Y. Fu, G.-H. Hou, J.-H. Xie, L. Xing, L.-X. Wang, Q.-L. Zhou, J. Org. Chem. 2004, 69, 8157-8160; d) H.-F. Duan, J.-H. Xie, W.-J. Shi, Q. Zhang, Q.-L. Zhou, Org. Lett. 2006, 8, 1479-1481.

[7] Nitrogen-based chelating ligands: a) C. Chen, S.-F. Zhu, X.-Y. Wu, Q.-L. Zhou, Tetrahedron: Asymmetry 2006, 17, 2761-2767; b) B. Liu, S.-F. Zhu, L.-X. Wang, Q.-L. Zhou, Tetrahedron: Asymmetry 2006, 17, 634641 ; c) Y.-Z. Zhang, S.-F. Zhu, L.-X. Wang, Q.-L. Zhou, Angew. Chem 2008, 120, 8624-8626; Angew. Chem. Int. Ed. 2008, 47, 8496-8498.

[8] P-N and P-O type chelating ligands: a) S.-F. Zhu, J.-B. Xie, Y.-Z. Zhang, S. Li, Q.-L. Zhou, J. Am. Chem. Soc. 2006, 128, 12886-12891; b) J.-B. Xie, J.-H. Xie, X.-Y. Liu, Q.-Q. Zhang, Q.-L. Zhou, Chem. Asian J. 2011, 6, 899-908; c) J.-H. Xie, X.-Y. Liu, J.-B. Xie, L.-X. Wang, Q.-L. Zhou, Angew. Chem. 2011, 123, 7467-7470; Angew. Chem. Int. Ed. 2011, 50, 7329-7332; d) S.-F. Zhu, Y.-B. Yu, S. Li, L.-X. Wang, Q.-L. Zhou, Angew. Chem. 2012, 124, 9002-9005; Angew. Chem. Int. Ed. 2012, 51 8872-8875; e) S. Yang, W. Che, H.-L. Wu, S.-F. Zhu, Q.-L. Zhou, Chem. Sci. 2017, 8, 1977-1980.

[9] J. Zheng, W.-J. Cui, C. Zheng, S.-L. You, J. Am. Chem. Soc. 2016, 138, 5242-5245.

[10] A chiral phosphine was used as a organocatalyst: Y. K. Chung, G. C Fu, Angew. Chem. 2009, 121, 2259-2261; Angew. Chem. Int. Ed. 2009, 48, 2225-2227.

[11] a) I. Čorić, S. Müller, B. List, J. Am. Chem. Soc. 2010, 132, 17370 17373; b) F. Xu, D. Huang, C. Han, W. Shen, X. Lin, Y. Wang, J. Org Chem. 2010, 75, 8677-8680; c) B. Xu, M.-L. Li, X.-D. Zuo, S.-F. Zhu Q.-L. Zhou, J. Am. Chem. Soc. 2015, 137, 8700-8703; d) S. Li, J.-W. Zhang, X.-L. Li, D.-J. Cheng, B. Tan, J. Am. Chem. Soc. 2016, 138, $16561-16566$.

[12] Although the pKa of 2 was estimated by DFT calculations, the synthesis has not been reported: C. Yang, X.-S. Xue, X. Li, J.-P. Cheng, J. Org Chem. 2014, 79, 4340-4351.

[13] K. Takahashi, Jpn. Kokai Tokkyo Koho, JP2008-262585, 2008

[14] (R)-SPINOL 3 is commercially available from Struchem, Daicel, and TCl. For synthesis and optical resolution, see: a) V. B. Birman, A. L. Rheingold, K.-C. Lam, Tetrahedron: Asymmetry 1999, 10, 125-131; b) J.-H. Zhang, J. Liao, X. Cui, K.-B. Yu, J. Zhu, J.-G. Deng, S.-F. Zhu, L.X. Wang, Q.-L. Zhou, L. W. Chung, T. Ye, Tetrahedron: Asymmetry 2002, 13, 1363-1366.

[15] M. Hirano, K. Osakada, H. Nohira, A. Miyashita, J. Org. Chem. 2002 67, 533-540.

[16] a) G. C. Lloyd-Jones, J. D. Moseley, J. S. Renny, Synthesis 2008, 661 689 ; b) J. N. Harvey, J. Jover, G. C. Lloyd-Jones, J. D. Moseley, P. Murray, J. S. Renny, Angew. Chem. 2009, 121, 7748-7751; Angew. Chem. Int. Ed. 2009, 48, 7612-7615; c) A. J. Perkowski, C. L. Cruz, D. A. Nicewicz, J. Am. Chem. Soc. 2015, 137, 15684-15687.

[17] Catalytic Asymmetric aminalization: a) G. B. Rowland, H. Zhang, E. B Rowland, S. Chennamadhavuni, Y. Wang, J. C. Antilla, J. Am. Chem. Soc. 2005, 127, 15696-15697; b) Y. Liang, E. B. Rowland, G. B. Rowland, J. A. Perman, J. C. Antilla, Chem. Commun. 2007, $4477-$ 4479; c) X. Cheng, S. Vellalath, R. Goddard, B. List, J. Am. Chem. Soc 
2008, 130, 15786-15787; d) S. Y. Park, Y. Liu, J. S. Oh, Y. K. Kweon, Y. B. Jeong, M. Duan, Y. Tan, J. W. Lee, H. Yan, C. E. Song, Chem. Eur. J. 2018, 24, 1020-1025.

[18] Although 2 showed better enantioselectivity than 1, we considered 1 would be suitable for this acid-base combined catalysis because 1 has two acidic functional groups. Therefore, 1 was chosen as a catalyst for further investigation.

[19] The effect of $\mathrm{Mg}^{2+}$ as a Lewis acid cannot be ruled out, but would be limited, because almost comparable results were obtained with other desiccants.
[20] When we performed a reaction for $20 \mathrm{~min}$ under the optimized conditions (Table 1, entry 14 ), 10 aa was obtained in $28 \%$ yield and $93 \%$ ee, indicating that no racemization of 10 aa occurred under these conditions.

[21] The optical rotation of $10 \mathbf{a b}$ was opposite to that of the (S)-isome reported in reference [3i]. Thus, the absolute configuration of $10 \mathrm{ab}$ was determined to be $(R)$. 
Entry for the Table of Contents (Please choose one layout)

\section{COMMUNICATION}

New chiral Brønsted acid catalysts with a spirocyclic backbone were developed. SPISA and its disulfonimide were synthesized from SPINOL using Pd-catalyzed NewmanKwart rearrangement as a key step, and evaluated in a catalytic asymmetric aminalization reaction. These new catalysts can provide a new good option for the development of asymmetric catalysis.
Takumaru Kurihara, Shun Satake, Manabu Hatano,Kazuaki Ishihara, Tatsuhiko Yoshino, * and Shigeki Matsunaga*

\section{Page No. - Page No.}

Synthesis of 1,1'-Spirobiindane-7,7'Disulfonic Acid and Disulfonimide: Application for Catalytic Asymmetric Aminalization 